Introduction

The commonest cause of hypogylycaemia in people with diabetes is the mismatch between insulin or oral hypoglycaemic drugs and carbohydrate intake or excercise. Hypoglycaemia in people without diabetes is usually due to reactive hyperinsulinaemia and can also result from severe illness, sepsis, malnutrition, Addison's disease, alcohol, inadvertent or facticious use of insulin or oral hypoglycaemic agents and islet cell tumous. The latter group occasionally poses a diagnostic challenge. We present a similar case where hypoglycaemia remained a mystery: an enigma entangled.

\section{The clinical case}

A 56 year old man was admitted from psychiatry ward after episode of symptomatic hypoglycaemia with capillary blood glucose of $2.5 \mathrm{mmol} / \mathrm{L}$. His background included chronic kidney disease on thrice weekly haemodialysis, ischaemic heart disease, cerebrovascular disease, hypertension and paranoid psychosis. He was inpatient in a psychiatric ward during the episode of hypoglycaemia. He was not known to have diabetes and denied taking hypoglycaemics. His oral intake was normal and weight was stable. He had another symptomatic fasting hypoglycamia after 22 hours of hospital admission with venous glucose of $1.5 \mathrm{mmol} / \mathrm{L}$. Simultaneous Insulin and C-peptide levels suggested endogenous source of hyperinsulinaemia. Biochemical investigations are summarised below.

\begin{tabular}{|l|l|l|}
\hline Investigation & Result & Normal limits \\
\hline Venous glucose & $1.5 \mathrm{mmol} / \mathrm{L}$ & \\
\hline Insulin & $320 \mathrm{mU} / \mathrm{L}$ & $3.0-17.0$ \\
\hline C peptide & $7935 \mathrm{pmol} / \mathrm{L}$ & $260-650$ \\
\hline Sulphonyluria screen & Negative & \\
\hline HbA1c & $29 \mathrm{mmol} / \mathrm{mol}$ & \\
\hline Chromagranin A & $260 \mathrm{pmol} / \mathrm{L}$ & $<60$ \\
\hline Chromogranin B & $202 \mathrm{pmol} / \mathrm{L}$ & $<150$ \\
\hline Short synacthen test & Normal & \\
\hline
\end{tabular}

Collateral history revealed complex psychosocial background surrounding the events. His psychiatric team confirmed that he had paranoid psychosis and believed that his family members were trying to kill him. Further information revealed that one of his close relatives had diabetes treated with metformin and repaglinide. $\mathrm{He}$ and his family denied drug dipensing error or mix up of tablets. His blood samples taken during hypoglycaemia were sent to the national laboratory for further drug screening to exclude inadvertent drug intake. High performance liquid chromatography (HPLC) detected his usual medications and metformin but no other oral hypoglycaemics. The laboratory also suggested that HPLC does not completely rule out presence of other drrugs as some of the drugs might not have been detected due to interaction of tubes containing gel in HPLC, although the possibilty of this was less likely.

Meanwhile, the medical team also suspected an insulinoma and booked CT abdomen which revealed a deformity in pancreatic head (see figures 1-4). MRI pancreas showed lobulation of pancreatic head with no foci of contrast enhancement. His case was discussed in pancreatic MDT meeting and the abnormality was thought to be an anatomical variation of pancreas rather than a tumour. The MDT recommended further investigations including endoscopic ultrasound
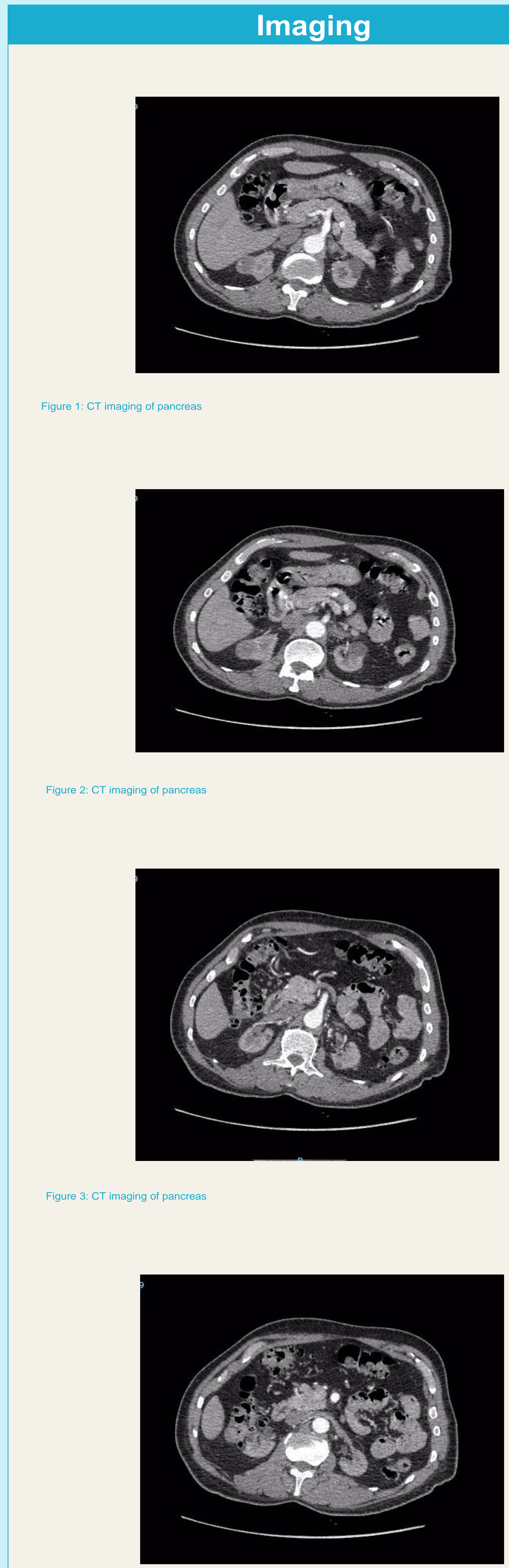

Chronic kidney disease poses a challenge in investigations for hypoglycaemia. Renal failure is a cause of hypoglycaemia and can falsely elevate chromogranin and C-peptide levels as they are excreted renally. Therefore, one should exercise caution when interpreting these results.

Hypoglycaemia due to sulphonylureas and repaglinide should always be considered in hypoglycaemia with high insulin and non suppressed C-peptide levels. Repaglinide is a prandial insulin secretagogue, and is primarily excreted in bile but clearance is reduced in advanced renal impairment (eGFR less than $30 \mathrm{~mL} / \mathrm{min}$ ). Therefore, prolonged monitoring of blood glucose is required in patients with suspected repaglinide intake. If sulphonyluria (and repaglinide) screen is negative, the endogenous source of hyperinsulinaemia should be sought. Surreptitious intake of insulin leads to hypoglycaemia with high insulin and suppressed C-peptide levels.

Irrespective of the cause of hypoglycaemia, patients should be counselled to identify symptoms of hypoglycaemia, measure capillary blood sugar and promptly treat hypoglycaemia. Long term follow up is essential. Hypoglycaemia may also have personal implications, e.g., driving and occupation.

\section{References}

\section{Choices, N. (2015) Hypoglycaemia (low blood sugar) - causes. Available at:
http://www.nhhs.uk/Conditions/Hypoglycaemia/Pages/Causes.aspx (Accessed: 31 October 2016}

Ltd, D. (2016) Repaglinide 0.5mg, 1mg, 2mg tablets - patient information leaflet (PIL) $-(e M C)$. Available
31 October 2016).

Schumacher S., Abbasi I., Weise D., Hatorp V., Sattler K., Sieber J., Hasslacher C. Single- and multiple-dose pharmacokinetics of repaglinide in patients with type 2
diabetes and renal impairment. Eur. J. Clin. Pharmacol. 2001;57:147-152. doi. 10.1007/s002280100280
1abetes and renal impant

\section{Further progress}

Patient did not comply with further investigations including an inpatient supervised fast and prolonged glucose tolerance test. Therefore, he was managed conservatively during hospital stay for 6 weeks and did not have further hypoglycaemic episodes. He and his family was educated for identification and management of hypoglycaemia. He was discharged with glucometer, oral dextrogel and glucagon injecton as required. He and his family did not report any hypoglycaemic events during his outpatient follow up for a year.

\section{Contact Information}

Contact : rahat.tauni@yahoo.co.uk or rahat.tauni@ldh.nhs.uk with any questions

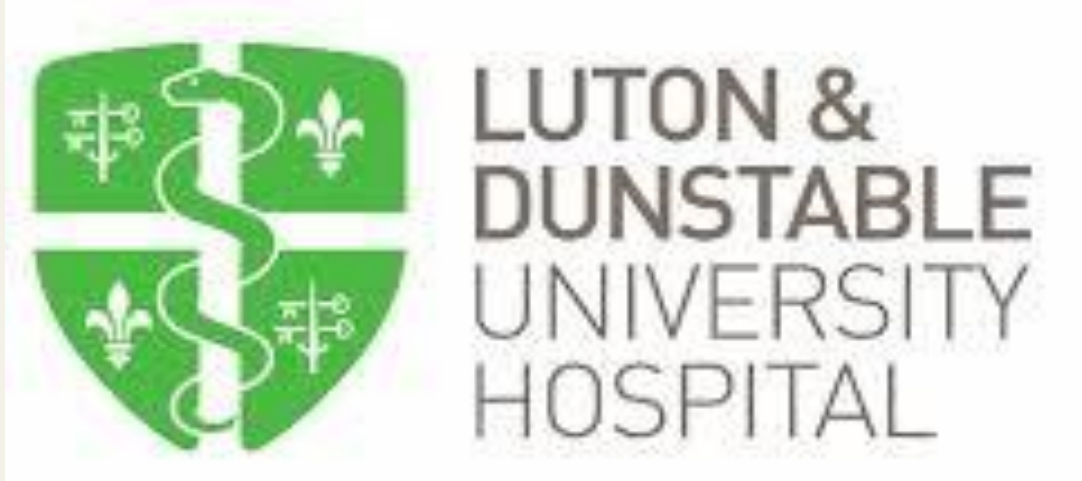

